\title{
Motivação e envolvimento acadêmico: um estudo com estudantes universitários
}

\author{
Rebeca Cruz Porto \\ Universidade Federal do Vale do São Francisco - Petrolina PE - Brasil \\ Marina Pereira Gonçalves \\ Universidade Federal do Vale do São Francisco - Petrolina - PE - Brasil
}

\begin{abstract}
Resumo
Pesquisas sugerem que motivação e envolvimento acadêmico sejam variáveis importantes para o contexto educacional. Assim, a presente pesquisa teve por objetivo principal investigar a relação entre estes construtos. A amostra foi composta por 406 estudantes universitários, a maioria (81,3\%) com idades variando entre 20 e 30 anos, do sexo feminino $(55,2 \%)$ e de instituições de ensino superior pública (70,4\%). Estes responderam a Escala de Motivação Acadêmica (EMA), a Escala de Envolvimento Acadêmico (EEA) e a questões sociodemográficas. Os resultados apontaram correlação positiva e significativa entre motivação e envolvimento acadêmico. Foram encontradas ainda diferenças em relação ao sexo, ao período e área do curso e ao envolvimento com atividades extracurriculares. Tais resultados foram discutidos à luz das teorias do envolvimento acadêmico e da autodeterminação. Sugere-se a realização de novas pesquisas a fim de melhor compreender as variáveis que influenciam na motivação e no envolvimento acadêmico de estudantes universitários.
\end{abstract}

Palavras-chave: Motivação; participação; Ensino Superior.

\section{Motivation and academic involvement: a study with university students}

\begin{abstract}
The researches suggest that motivation and academic involvement are important variables for the educational context. Thus, the main goal of this research was to investigate the relationship between these constructs. The sample consisted of 406 university students, the majority (81.3\%) with ages varying between 20 and 30 years, female $(55.2 \%)$ and public higher education institutions $(70.4 \%)$. These answered the Academic Motivation Scale (EMA), the Academic Involvement Scale (EEA) and sociodemographic issues. The results showed a positive and significant correlation between motivation and academic involvement. Differences were also found regarding the sex, the period and area of the course and the involvement with extracurricular activities. These results were discussed in the light of theories of academic involvement and selfdetermination. It is suggested to carry out new research in order to better understand the variables that influence the motivation and academic involvement of university students.
\end{abstract}

Keywords: Motivation; participation; Higher education.

\section{Motivación y participación académica: estudio con estudiantes universitarios}

\section{Resumen}

Investigaciones sugieren que motivación y participación académica sean variables importantes para el contexto educacional. Así, en la presente investigación se tuvo el objetivo principal investigar la relación entre estos constructos. El muestreo fue compuesto por 406 estudiantes universitarios, la mayor parte (el $81,3 \%$ ) con edades variando entre 20 y 30 años, del sexo femenino (el $55,2 \%$ ) y de instituciones de enseñanza universitaria pública (el 70,4\%). Estos respondieron la Escala de Motivación Académica (EMA), la Escala de Participación Académica (EEA) y las cuestiones sociodemográficas. Los resultados enseñan correlación positiva y significativa entre motivación y participación académica. Se encontraron aun diferencias en relación al sexo, al período y área del curso y a la participación con actividades extracurriculares. Se discutieron los resultados a la luz de las teorías de la participación académica y de la autodeterminación. Se sugiere la realización de nuevas investigaciones con la finalidad comprender mejor las variables que influencian en la motivación y en la participación académica de estudiantes universitarios.

Palabras clave: Motivación; Participación; Enseñanza Universitaria. 


\section{Introdução}

O número de estudantes matriculados na educação superior no Brasil tem aumentado significativamente (Corbucci, Kubota, \& Meira, 2016; Traina-Chacon \& Calderón, 2015); apenas entre 2003 e 2013 o aumento foi de 76,4\% (Instituto Nacional de Estudos e Pesquisas Educacionais [INEP], 2014). No entanto, algumas dificuldades educacionais a exemplo do fracasso escolar, da evasão e o insucesso acadêmico têm preocupado os educadores (Da-Veiga-Simão, Bragagnolo-Frison, \& Rodrigues-Nonticuri, 2015), dificuldades estas que podem estar relacionadas direta ou indiretamente com a motivação para a aprendizagem e com o envolvimento acadêmico (Mercuri \& Fior, 2012; Corbucci \& cols., 2016; Davoglio, Santos, \& Lettnin, 2016).

Diante do exposto, verifica-se a necessidade de estudos que visem conhecer o perfil do estudante de ensino superior no Brasil, sobretudo no que diz respeito ao envolvimento acadêmico e à motivação, uma vez que pesquisas desta natureza ainda são escassas em nível nacional (Fior, Mercuri, \& Silva, 2013). Desse modo, a presente pesquisa se propõe a investigar a motivação e o envolvimento acadêmico de estudantes universitários.

\section{Motivação Acadêmica}

A motivação é um estado em que uma pessoa é movida a fazer algo, e com base na Teoria da Autodeterminação, ela pode ser diferenciada tanto em relação ao nível (muita ou pouca motivação) quanto à orientação (motivação intrínseca ou motivação extrínseca). A motivação intrínseca é o fazer algo simplesmente pelo prazer de fazer, sendo ela a base enérgica que move uma pessoa a agir em busca de uma satisfação pessoal; já a motivação extrínseca envolve um resultado separado da própria ação, que seria a busca por recompensas ou a esquiva de punições (Ryan \& Deci, 2000; Deci \& Ryan, 2012).

Diante de uma série de teorias sobre motivação e, especificamente, sobre motivação acadêmica, na presente pesquisa será levada em consideração a Teoria da Autodeterminação (TAD) de Ryan e Deci (2000) por apresentar relativa importância na compreensão desse construto, sobretudo no contexto educacional. Esta teoria propõe que todas as pessoas apresentam uma propensão inata para desenvolver sua própria determinação e que se envolvem em atividades que Ihes possibilitem a satisfação de necessidades psicológicas básicas: competência, autonomia e relacionamento. Essa teoria apresenta um continuum de desenvolvimento da autodeterminação (Vansteenkiste, Lens, \& Deci, 2006; Deci \& Ryan, 2012), subdividida em três categorias: 1) Desmotivação, 2) Regulações por motivação extrínseca (regulação externa, regulação introjetada, regulação identificada e regulação integrada) e 3) Motivação intrínseca.

A desmotivação é um estado no qual as pessoas não têm a intenção de se comportar. Já a regulação externa é a forma mais altamente controlada e menos autônoma de motivação, nessa subcategoria o comportamento é controlado por agentes externos ao próprio comportamento, ou seja, o indivíduo se comporta para alcançar recompensas ou evitar punições (Deci \& Ryan, 2000; Deci \& Ryan, 2012; Vansteenkiste, Lens, \& Deci, 2006). À medida que valores e regulações comportamentais são introjetados no indivíduo, este os internaliza; desta forma, as consequências são mais contingentes ao comportamento, este fenômeno é denominado pela TAD como regulação introjetada, e neste tipo as consequências são administradas pelos próprios indivíduos (Deci \& Ryan, 2000; Deci \& Ryan, 2012). Por sua vez, regulação identificada consiste num processo através do qual as pessoas reconhecem e aceitam a relevância pessoal da atividade por si mesma. Ao se identificarem com o valor da atividade as pessoas têm a sua internalização mais regulamentada, aceitam-na melhor e a exercem voluntariamente como sua (Deci \& Ryan, 2000; Deci \& Ryan, 2012; Vansteenkiste \& cols., 2006). Finalmente, a regulação integrada é vista como a forma de motivação extrínseca mais internalizada, aqui ela se torna congruente com outras identificações, necessidades e experiências envolvendo tanto a importância do comportamento para a pessoa quanto uma identificação com aspectos do próprio eu (Deci \& Ryan, 2000; Deci \& Ryan, 2012).

Por outro lado, a motivação intrínseca, que é a forma mais autodeterminada de motivação e a mais autônoma do continuum da TAD, é definida pela postura do indivíduo diante da tarefa; nesse tipo de motivação, a ação independe de consequências externas, pelo contrário, o que move o sujeito é a tarefa em si mesma, a satisfação em realizá-la; esta forma de motivação tem sido relacionada com saúde mental e melhores desempenhos em geral (Ryan \& Deci, 2000; Deci \& Ryan, 2012).

Dentro do contexto educacional, Deci e Ryan (2012) apontam que a motivação intrínseca é um melhor preditor para o engajamento do estudante na tarefa, enquanto que as regulações integrada e identificada são melhores preditores de esforço na tarefa.

Nessa linha algumas pesquisas vêm sendo realizadas. Por exemplo, Leal, Miranda e Carmo (2013) realizaram um estudo no qual encontraram que no início do curso os estudantes têm uma motivação mais autônoma (intrínseca, integrada e identificada) e os concluintes uma motivação não autônoma (introjetada, externa e desmotivada); para esses autores a inversão acontece processualmente, ao longo do curso. Em comparação entre instituições públicas e privadas Almeida e Rufini (2012) encontraram que, em estudantes de instituições privadas, as médias dos fatores de motivação extrínseca por regulação introjetada, identificada, integrada e motivação intrínseca foram superiores às obtidas por participantes da rede pública, e embora com diferença mínima, o resultado para a desmotivação em alunos de instituições públicas foi maior do que em alunos da rede privada.

Ainda baseado na TAD, Joly e Prates (2011), Almeida e Ruffini (2012), Leal e cols. (2013), e Davoglio e cols. (2016) realizaram estudos que apontam para um perfil autodeterminado dos universitários incluídos na amostra. 


\section{Envolvimento acadêmico}

Na literatura internacional o envolvimento acadêmico vem sendo estudado há muito tempo, sendo Astin (1984) o pioneiro destes estudos. Entretanto, nos últimos anos, um termo semelhante tem emergido em algumas pesquisas, o engajamento; eles não apresentam exatamente o mesmo significado, entretanto, ambos compreendem que a aprendizagem e o desenvolvimento do estudante estão relacionados com participação nas atividades acadêmicas, tanto dentro como fora da sala de aula, e que estão relacionados a consequências educacionais desejáveis (Fior \& cols., 2013).

Segundo Eccles e Wang (2012), o engajamento é atualmente um dos tópicos mais discutidos no que diz respeito à realização escolar e visa a contribuir para que as instituições possam criar ambientes propícios para aprendizagens mais eficazes (Astin, 1984; Eccles \& Wang, 2012; Reeve, 2012).

De maneira mais pontual, a literatura aponta que o envolvimento do estudante nas diversas experiências disponibilizadas pela instituição é uma das variáveis importantes na predição do rendimento acadêmico e da permanência no ensino superior (Fior \& cols., 2013).

Para Astin (1984), o envolvimento é um termo ativo que implica um componente comportamental, sendo o envolvimento acadêmico definido pelo autor como a quantidade de energia física e psicológica que o aluno dedica à experiência acadêmica. Quanto mais tempo o estudante dedica às atividades na universidade, tanto dentro quanto fora da sala de aula, maior é o envolvimento acadêmico desse estudante e, consequentemente, maior o valor da aprendizagem e desenvolvimento pessoal.

Nesta mesma direção, Fior e cols. (2013) em um estudo com 1070 estudantes frequentando até o segundo semestre verificaram que tanto atividades curriculares quanto atividades extracurriculares produzem envolvimento acadêmico nos estudantes. Por sua vez, Costa, Araújo e Almeida (2014) encontraram uma correlação positiva e estatisticamente significativa entre envolvimento e rendimento acadêmico.

Por outro lado, Mercuri e Fior (2012) mostram que estudantes com menor participação em atividades obrigatórias e em atividades não obrigatórias apresentam um risco maior de abandonar o curso do que estudantes envolvidos, sugerindo assim que o envolvimento dos estudantes nestas atividades reduz a probabilidade de evasão. Sendo assim, entendemos que há uma relação bidirecional entre a motivação e o envolvimento uma vez que a motivação influencia o estudante a se envolver mais com as tarefas (Leal \& cols., 2013) e este engajamento produz mudanças motivacionais (Reeve, 2012). Neste sentido, Santos, Mognon, Lima e Cunha (2011) realizaram uma pesquisa visando identificar possíveis relações entre a motivação e a vida acadêmica e encontraram diferenças estatisticamente significativas entre envolvimento não obrigatório e motivação para a aprendizagem em relação a diferentes cursos, de diferentes áreas de conhecimento.
Diante do exposto, o presente estudo se propôs a verificar a correlação entre motivação e envolvimento acadêmico, uma vez que este modelo explicativo é novo e um pouco controverso (Reeve, 2012). Especificamente, buscar-se-á avaliar o nível de motivação e de envolvimento acadêmico de estudantes universitários. Será verificado ainda se há diferença motivacional e de envolvimento em relação ao sexo, à participação em atividades extracurriculares, em função do tipo de Instituição (pública ou privada) e se há períodos do curso (início, meio e/ou final) em que os estudantes estejam mais motivados e envolvidos.

\section{Método}

\section{Amostra}

Participaram desta pesquisa 406 estudantes universitários da região do Vale do São Francisco (PE e BA), sendo a maioria de instituição de ensino superior (IES) pública $(70,4 \%)$, seguidos de $29,6 \%$ de instituições privadas, a maioria (81,3\%) com idades entre 20 e 30 anos, distribuídos equitativamente quanto ao sexo $(55,2 \%$ do sexo feminino; $44,3 \%$ do sexo masculino e $0,5 \%$ transgênero). No que se refere às áreas de conhecimento, participaram: 46,6\% de Ciências Humanas; $29,1 \%$ de Ciências Exatas e 24,4\% de Ciências da Vida. A maior parte deles (45,6\%) indicou estar no meio do curso (entre $4^{\mathrm{a}}$ e $8^{\circ}$ período), seguidos de $33,7 \%$ do final de curso (sendo $9^{\circ}$ e $10^{\circ}$ período) e $20,7 \%$ de início de curso ( $1^{\circ}$ ao $3^{\circ}$ período). $62,3 \%$ dos pesquisados afirmaram participar de, ao menos, uma atividade extracurricular e $37,7 \%$ indicaram não participar de nenhuma atividade extracurricular.

Tratou-se, portanto, de uma amostra selecionada por conveniência, sendo incluídos no estudo aqueles que ainda se encontravam na graduação e que fossem estudantes de uma IES da região do Vale do São Francisco.

\section{Instrumentos}

- Escala de Motivação Acadêmica (ECA): Validada para o contexto brasileiro por Guimarães e Bzuneck (2008), possui sete subescalas referentes ao continuum da autodeterminação da TAD, distribuídas em 31 itens respondidos por meio de uma escala de resposta do tipo Likert de sete pontos, variando de $1=$ Nenhuma correspondência a $7=$ Total correspondência. Esta medida apresenta índices de consistência interna satisfatórios, de acordo com os autores, com alfas de Cronbach variando de 0,61 a 0,79.

- Escala de Envolvimento Acadêmico (EEA): Construída e validada para o contexto brasileiro por Fior e cols. (2013), possui 23 itens, distribuídos em duas subescalas: envolvimento em atividades obrigatórias $(\alpha=0,85)$ e envolvimento em atividades não obrigatórias $(\alpha=0,73)$, respon- 
didos em uma escala Likert de cinco pontos $(1=$ Discordo totalmente a 5 = Concordo totalmente).

- Questões sociodemográficas: Os participantes responderam ainda questões como idade, sexo, estado civil, dentre outras informações que permitissem identificar o perfil dos estudantes pesquisados.

\section{Procedimento}

Os dados foram coletados a partir de uma plataforma online, o google docs. O link para acessar o questionário da pesquisa foi enviado para e-mails institucionais de IES públicas e privadas da região do Vale do São Francisco (PE e BA). Os participantes também foram abordados através de páginas de redes sociais das instituições envolvidas na pesquisa. Inicialmente, na primeira página da pesquisa, o participante tinha acesso ao Termo de Consentimento Livre e Esclarecido, que descrevia os objetivos da pesquisa, bem como seu caráter voluntário e a confidencialidade de suas respostas. Ao final do questionário, o participante clicava em "enviar formulário", e as pesquisadoras recebiam as respostas em um banco de dados. Esta pesquisa foi aprovada pelo comitê de Ética da Universidade Federal do Vale do São Francisco, sob o $n^{\circ} 1.018 .739$.

\section{Análise dos dados}

Os dados obtidos foram analisados por meio do Pacote Estatístico para as Ciências Sociais (SPSS versão 20). Foram realizadas estatísticas descritivas (medidas de tendência central e dispersão) para caracterizar a amostra; correlações $r$ de Pearson para verificar a relação entre motivação e envolvimento acadêmico; e ainda, análises de variância (ANOVA e MANOVA) para verificar diferenças entre os construtos investigados em função do sexo, idade, período do curso e envolvimento com atividades extracurriculares.

\section{Resultados}

A fim de cumprir com o objetivo principal desta pesquisa, procedeu-se inicialmente com uma análise de Correlação $r$ de Pearson, como pode ser visto na Tabela 1.

Tabela 1. Correlação entre motivação acadêmica e envolvimento acadêmico.

ENVOLVIMENTO ACADÊMICO

\begin{tabular}{lcc} 
MOTIVAÇÃO ACADÊMICA & Envolvimento Obrigatório & Envolvimento não obrigatório \\
\hline Desmotivação & $-0,330^{* * *}$ & $-0,294^{* * *}$ \\
Reg. Ext. por freq. & $-0,265^{* * *}$ & $-0,284^{* * *}$ \\
Reg. Ext. por rec.social & $-0,152^{* *}$ & $-0,082$ \\
Regulação introjetada & 0,008 & $-0,072$ \\
Regulação identificada & $0,223^{* *}$ & $0,110^{*}$ \\
Regulação integrada & $0,302^{* * *}$ & $0,164^{* *}$ \\
Motivação intrínseca & $0,403^{* * *}$ & $0,386^{* * *}$
\end{tabular}

Nota: diferença considerada estatisticamente significativa: ${ }^{*} p<0,05,{ }^{* *} p<0,01,{ }^{* * *} p<0$,

Tabela 2. Média e desvios padrões dos participantes na avaliação da Motivação e Envolvimento acadêmico.

\begin{tabular}{|c|c|c|c|c|c|}
\hline Variáveis & $\mathrm{N}$ & Mínimo & Máximo & Média & DP \\
\hline Desmotivação & 406 & 1 & 7 & 1,72 & 1,064 \\
\hline Regulação Externa por frequência às aulas & 406 & 1 & 7 & 3,50 & 1,408 \\
\hline Regulação Externa por recompensas sociais & 406 & 1 & 6 & 2,07 & 1,045 \\
\hline Regulação Introjetada & 406 & 1 & 7 & 3,97 & 1,429 \\
\hline Regulação Identificada & 406 & 1 & 7 & 3,99 & 1,744 \\
\hline Regulação Integrada & 406 & 1 & 7 & 5,74 & 1,021 \\
\hline Motivação Intrínseca & 406 & 1 & 7 & 4,79 & 1,483 \\
\hline Atividades Obrigatórias & 406 & 2 & 5 & 3,68 & 0,447 \\
\hline Atividades não obrigatórias & 406 & 1 & 5 & 2,91 & 0,710 \\
\hline
\end{tabular}


Como indicado na Tabela 1, observaram-se correlações negativas e significativas entre a desmotivação e o envolvimento (com atividades obrigatórias e com atividades não obrigatórias); por outro lado, houve correlação positiva e significativa da motivação por regulação integrada e da motivação intrínseca com ambos os tipos de envolvimento: obrigatório e não obrigatório.

A fim de responder aos demais objetivos desta pesquisa, foi verificada a pontuação média dos participantes nas variáveis motivação e envolvimento acadêmico, considerando o ponto médio da escala de resposta dos instrumentos, sendo a de motivação de 1 a 7 pontos e a de envolvimento acadêmico de 1 a 5 pontos, como mostra a Tabela 2 .

Como pode ser observado, os estudantes apresentaram maiores médias na regulação integrada, seguidas de motivação intrínseca e regulação identificada, respectivamente, e as menores médias foram vistas em desmotivação, seguidas de regulação externa por recompensas sociais.

Dando continuidade, para averiguar possíveis diferenças significativas entre os grupos estudados, procedeu-se com uma análise de variância multivariada (MANOVA), como indicada na Tabela 3. De modo geral, foi possível concluir que houve efeito multivariado entre motivação e envolvimento acadêmico no que se refere ao tipo de instituição, à área do curso, aos tipos de atividades e ao período do curso $[\lambda(9,336)=0,41 ; p<0,001)]$.

Especificamente quanto ao tipo de instituição, foi possível verificar que os participantes da IES privada apontaram maior média $(m=4,51 ; d p=0,16)$ do que aqueles de IES pública ( $m=3,81 ; d p=0,11)$ no fator regulação introjetada, sendo este resultado estatisticamente significativo $[F(1,406)$ $=6,705 ; p<0,01]$; e no fator motivação intrínseca os participantes de IES privadas também apresentaram maior média ( $m=5,23 ; d p=0,19)$ do que os das IES públicas $(m=4,73$; $d p=0,12)$, sendo este resultado também estatisticamente significativo $[F(1,406)=5,299 ; p<0,02]$.

Em relação à participação em atividades extracurriculares, os estudantes que participavam de atividades extraclasse apresentaram maior média $(m=1,89 ; d p=0,11)$ em desmotivação do que aqueles que não participavam ( $m=1,54 ; d p=0,10)$, sendo este resultado estatisticamente significativo $[F(1,406)=4,897 ; p=0,028]$. No entanto, os estudantes que participavam de atividades extraclasse obtiveram maior média ( $m=4,22 ; d p=0,16)$ no fator regulação identificada quando comparados com aqueles que não participavam desde tipo de atividade $(m=3,87 ; d p=0,18)$, também este resultado sendo estatisticamente significativo $[F(1,406)=7,269 ; p=0,007]$.

Tabela 3. Diferenças de média da Motivação e Envolvimento acadêmico em função das variáveis tipo de IES, sexo, área do curso, tipo de atividades e período do curso.

\begin{tabular}{llllll}
\hline & \multicolumn{1}{c}{ IES (I) } & \multicolumn{1}{c}{$\begin{array}{c}\text { Atividade } \\
\text { (Atv. })\end{array}$} & Área (A) & Sexo (S) & Período (I) \\
\hline Desmotivação & $F(1,406)$ & $F(1,406)^{*}$ & $F(1,406)$ & $F(1,406)$ & $F(1,406)^{*}$ \\
& 1,15 & 4,89 & 0,62 & 0,63 & 3,27 \\
Ext. Freq & $F(1,406)$ & $F(1,406)$ & $F(1,406)$ & $F(1,406)$ & $F(1,406)$ \\
& 2,05 & 0,24 & 0,72 & 1,69 & 0,09 \\
Ext. Soc & $F(1,406)$ & $F(1,406)$ & $F(1,406)$ & $F(1,406)$ & $F(1,406)$ \\
& 0,08 & 1,40 & 0,82 & 1,14 & 0,13 \\
Introjetada & $F(1,406)^{*}$ & $F(1,406)$ & $F(1,406)$ & $F(1,406)$ & $F(1,406)$ \\
& 6,70 & 0,006 & 0,11 & 1,67 & 1,41 \\
Identificada & $F(1,406)$ & $F(1,406)^{* *}$ & $F(1,406)$ & $F(1,406)$ & $F(1,406)$ \\
& 2,19 & 7,26 & 0,24 & 1,78 & 0,17 \\
Integrada & $F(1,406)$ & $F(1,406)$ & $F(1,406)$ & $F(1,406)$ & $F(1,406)$ \\
& 0,07 & 2,23 & 0,50 & 0,92 & 0,45 \\
Intrínseca & $F(1,406)^{*}$ & $F(1,406)$ & $F(1,406)$ & $F(1,406)$ & $F(1,406)$ \\
& 5,29 & 2,40 & 0,926 & 0,86 & 2,40 \\
\hline Env. Obr & $F(1,406)$ & $F(1,406)$ & $F(1,406)^{* *}$ & $F(1,406)$ & $F(1,406)$ \\
& 2,72 & 1,17 & 5,40 & 1,05 & 0,93 \\
Env. N. Obr & $F(1,406)$ & $F(1,406)$ & $F(1,406) * *$ & $F(1,406)$ & $F(1,406)$ \\
& 0,10 & 1,32 & 7,32 & 0,25 & 0,24 \\
\hline
\end{tabular}

Nota: diferença considerada estatisticamente significativa: ${ }^{*} p<0,05,{ }^{* *} p<0,01,{ }^{* * *} p<0,001$. 
No que diz respeito à área do curso o teste post hoc Bonferroni indicou que os estudantes da área de Humanas apresentam maior motivação intrínseca do que aqueles da área de Exatas ( $m=0,49 ; d p=0,17 ; p<0,01)$. Já em relação ao envolvimento acadêmico com atividades obrigatórias, estudantes da área de ciências da Vida apresentam maior envolvimento em atividades obrigatórias do que aqueles das áreas de Exatas ( $m=0,17 ; d p=0,06 ; p<0,001$ ) e de Humanas ( $m=0,16$; $d p=0,05 ; p<0,01$ ). Quanto ao fator envolvimento em atividades não obrigatórias, ocorreu o mesmo, uma vez que os estudantes de ciências da Vida também apresentaram maior média do que os estudantes das ciências Humanas ( $m=0,38 ; d p=0,08 ; p<0,001$ ) e das ciências Exatas ( $m=0,50 ; d p=0,09 ; p<0,001)$.

No que se refere à variável sexo, também a partir do post hoc Bonferroni, foi possível verificar que as mulheres pontuaram mais do que os homens na motivação por regulação identificada ( $m=0,47 ; d p=0,17 ; p<0,02)$ e na regulação integrada $(m=0,32 ; d p=0,10 ; p<0,01)$. Quanto ao envolvimento acadêmico, só houve diferença quanto ao envolvimento com atividades obrigatórias, verificando-se que as mulheres pontuaram mais $(m=0,17 ; d p=0,04 ; p$ $<0,001$ ), não havendo possibilidade de comparação com os estudantes transgêneros pela baixa representatividade deste grupo nesta pesquisa.

Finalmente, quanto ao período do curso, também após um post hoc Bonferroni, foi verificado que no tipo de motivação por regulação introjetada os alunos do início do curso apresentaram maior média quando comparados com os do meio ( $m=0,56 ; d p=0,18 ; p<0,01)$ e do final do curso ( $m=0,55 ; d p=0,19 ; p<0,01)$. O mesmo foi verificado na motivação por regulação identificada, onde os discentes do início do curso obtiveram maior média do que os do meio $(\mathrm{m}$ $=0,75 ; d p=0,22 ; p<0,002)$ e do final do curso $(m=0,91 ; d p$ $=0,91 ; p<0,001)$. Houve ainda diferença significativa quanto à regulação integrada, ocorrendo, neste caso, maiores médias apenas entre os estudantes do início do curso em relação aos do final do curso ( $m=0,38 ; d p=0,14 ; p<0,02)$.

Por sua vez, na motivação intrínseca foram encontradas maiores diferenças de médias entre os estudantes do início $(m=0,84 ; d p=0,20 ; p<0,001)$ e do final do curso $(m$ $=0,84 ; d p=0,20 ; p<0,001)$, seguidas das diferenças entre início $(m=0,84 ; d p=0,20 ; p<0,001)$ e meio de curso $(m$ $=0,64 ; d p=0,19 ; p<0,003)$. Quanto ao envolvimento acadêmico, só foi possível verificar diferença significativa em relação ao envolvimento com atividades obrigatórias, onde os estudantes do início do curso apresentaram maior média quando comparados àqueles do final do curso $(m=0,20 ; d p$ $=0,06 ; p<0,003$ ), e também foi observado que os de meio do curso apresentaram maiores médias em relação aos do final do curso $(m=0,12 ; d p=0,05 ; p<0,04)$.

\section{Discussão}

Respondendo aos objetivos propostos neste trabaIho, verificaram-se correlações significativas entre motiva- ção e envolvimento acadêmico corroborando com alguns outros estudos na área (Santos \& cols., 2011). Dentre estas correlações destacamos as correlações positivas e significativas entre a motivação intrínseca e o envolvimento tanto obrigatório como não obrigatório, um dado positivo e até esperado, uma vez que em pesquisas educacionais a motivação intrínseca tem sido relacionada a maior engajamento do estudante com suas experiências (Deci \& Ryan, 2012).

Com relação ao perfil dos estudantes universitários, os dados os apontam como motivados na forma autodeterminada, o que significa que estão motivados para realizarem as tarefas acadêmicas visando ganhos pessoais, mas também sentem prazer em realizá-las (Leal \& cols., 2013), corroborando com dados encontrados por Joly e Prates (2011), Almeida e Ruffini (2012), Leal e cols. (2013).

Outra característica dos estudantes dessa amostra foi o maior envolvimento em atividades obrigatórias, o que é esperado, visto que estas atividades, como o próprio nome sugere, são obrigatórias e essenciais para a conclusão do curso e para formação profissional do estudante. Todavia, notamos também que o envolvimento com atividades não obrigatórias ficou acima da média, um resultado positivo, visto que tais atividades como iniciação científica, monitoria e extensão, dentre outras, são essenciais para uma formação mais completa (Silva, Melo, Nunes, \& Moura, 2012), possibilitando ao estudante envolver-se em atividades que mais lhe apetecem, além de escolher aquelas que melhor compõem seu currículo.

Em relação às variáveis: tipo de instituição, área do curso, atividades extracurriculares, sexo e período do curso, foi observado efeito multivariado entre estas e a motivação e envolvimento acadêmico. Quanto ao tipo de instituição, os estudantes de IES privada apresentaram maior motivação do que os de IES pública, assim como os estudantes da amostra de Almeida e Rufini (2012). Este resultado pode ter ocorrido devido ao fato de que durante a realização da pesquisa algumas das IES públicas estavam repondo o calendário acadêmico após um período extenso de greve, algo que não ocorrera nas instituições privadas. Entretanto, esta diferença de motivação entre estudantes de IES pública e privada carece de futuros estudos para melhor compreendê-los.

Outra variável analisada na pesquisa foi a participação em atividades extracurriculares. Os resultados apontaram que estudantes que participam de atividades extracurriculares são mais motivados na forma identificada, na qual o comportamento é reconhecido e valorizado pelo próprio indivíduo (Deci \& Ryan, 2012); resultados semelhantes foram encontrados por Almeida e Rufini (2012). Verificou-se também que no fator desmotivação houve diferença significativa com média superior aos participantes de atividades extracurriculares. Devemos salientar aqui que, apesar dessa diferença significativa, a média geral da amostra no fator desmotivação foi bem abaixo da média quando comparada aos demais fatores; entretanto, dentro dessa amostra, os estudantes que participam das atividades extraclasses estariam mais desmotivados. No entanto, faz-se necessário que novas pesquisas sejam desenvolvidas nesse âmbito, a 
fim de averiguar quais fatores influenciam na motivação e na desmotivação tanto dos participantes de atividades extraclasses como de não participantes.

No que diz respeito à área do curso, os dados indicam que os estudantes da área de humanas apresentam maior motivação do que aqueles da área de exatas. Entre ciências humanas e ciências da vida não foram encontradas diferenças significativas, corroborando com os estudos de Joly e Prates (2011) e Almeida e Rufini (2012), que também não encontraram diferenças significativas entre estudantes de ciências da vida e humanas. Sugere-se que essa diferença na média motivacional se dê por conta das altas taxas de reprovação nos cursos de exatas, principalmente nos períodos iniciais, nas disciplinas de cálculo (Rodrigues \& cols., 2012), que podem interferir na motivação dos estudantes. Já em relação ao envolvimento, os resultados apontam que os estudantes de ciências da vida dessa amostra são mais envolvidos tanto em atividades obrigatórias quanto em atividades não obrigatórias do que estudantes de ciências exatas e de ciências humanas.

No que se refere ao sexo, as mulheres apresentaram pontuação mais alta que os homens nos fatores motivação por regulação identificada e por regulação integrada, divergindo de outros estudos como Joly e Prates (2011) e Davoglio e cols. (2016) que não encontraram diferenças significativas quanto a essa variável. Ainda em relação ao sexo, foram verificadas diferenças significativas entre homens e mulheres no fator envolvimento com atividades obrigatórias, sendo que as mulheres apresentaram uma média mais elevada, corroborando com a hipótese de correlação entre motivação e envolvimento (Leal \& cols., 2013; Reeve, 2012), uma vez que as mulheres apresentaram maior escore tanto para motivação quanto para envolvimento acadêmico.

Finalmente, observou-se neste estudo que os estudantes de início de curso apresentaram maior motivação que estudantes de meio e fim de curso, em especial no fator motivação intrínseca, corroborando com Leal e cols. (2013), dado que pode ser apoiado na ideia de que por serem ingressantes chegam à universidade carregados de expectativas positivas acerca do novo universo e do fato de estarem iniciando uma formação de nível superior, o que corresponde a uma perspectiva de futuro profissional mais concreta e promissora. Ademais, verificou-se ainda que os estudantes do início do curso são mais envolvidos com atividades obrigatórias do que os estudantes em final de curso, sendo possível inferir que estudantes de início de curso não tenham tido ainda oportunidades de participar de atividades extraclasses, uma vez que estas vão aparecendo ao longo do curso, como possibilidade de participação em grupos de pesquisa, extensão, monitorias etc., e neste sentido acabam ficando mais envolvidos com as atividades obrigatórias.

\section{Considerações Finais}

Os resultados principais desta pesquisa foram alcançados, porém algumas limitações podem ser apontadas como, por exemplo, o fato de ter sido realizada uma coleta de forma online, onde o controle sob as condições que os sujeitos responderam a pesquisa são desconhecidas. Não foi possível fazer comparações entre alguns grupos específicos, como instituições de ensino privado por área, por conta do baixo número de respostas de estudantes de IES particular. Também não foi possível verificar e especificar quais tipos de atividades extracurriculares os estudantes mais participam.

Apesar de tais limitações, a presente pesquisa trouxe algumas contribuições no que diz respeito à compreensão acerca da motivação e do envolvimento acadêmico de estudantes do ensino superior no Brasil, especificamente em cidade do interior de PE e BA. Entretanto, sugere-se que em estudos futuros sejam realizadas pesquisas que investiguem as diferentes formas de ensino superior, como a licenciatura e os ensinos técnicos, nas modalidades semipresenciais e à distância (EAD), bem como diferenciar os tipos de motivação e envolvimento acadêmico entre essas modalidades. Do mesmo modo, é necessário que se amplie o leque de pesquisas sobre a motivação e o envolvimento acadêmico por áreas de curso, buscando, assim, conhecer fatores que possam ajudar no engajamento dos estudantes.

Por fim, espera-se que os resultados aqui encontrados possam subsidiar possíveis estratégias de intervenção capazes de reduzir a evasão e o insucesso acadêmico de estudantes universitários brasileiros.

\section{Referências}

Almeida, M.S. \& Rufini, S.É. (2012). A motivação para aprender no ensino superior [Resumo]. Em IX Seminário de pesquisa em Educação da Região Sul (Org.), Anais do IX Seminário de pesquisa em Educação da Região Sul, Caxias do Sul - RS: ANPED SUL.

Astin, A.W. (1984). Student involvement: a developmental theory for higher education. Journal of College Student Personnel, 25 (1), 297-308.

Costa, A.R., Araújo, A.M., \& Almeida, L.S. (2014). Envolvimento académico de Estudantes de Engenharia: Contributos para a validação Interna e Externa de uma escala de Avaliação. Revista E-Psi, 4 (1), 142-155.

Corbucci, P.R., Kubota, L.C., \& Meira, A.P.B. (2016). Evolução da educação superior privada no Brasil: da reforma universitária de 1968 à década de 2010. Radar, 46, 7-12.

Da-Veiga-Simão, A.M., Bragagnolo-Frison, L.M., \& RodriguesNonticuri, A. (2015). Dos significados à autorregulação: perspectivas de estudantes com trajetórias acadêmicas de insucesso. Revista de Estudios e Investigación en Psicología y Educación, (01), 048-053.

Davoglio, T.R., Santos, B.S., \& Lettnin, C.C. (2016). Validação da Escala de Motivação Acadêmica em universitários brasileiros. Ensaio: Avaliação e Políticas Públicas em Educação, 24(92), 522-545. 
Deci, E.L. \& Ryan. R.M. (2000). The "What" and "Why" of Goal Pursuits: Human Needs and the Self-Determination of Behavior. Psychological Inquiry, 11 (4), 227-268.

Deci, E.L. \& Ryan, R.M. (2012). Motivation, personality, and development within embedded social contexts: An overview of selfdetermination theory. The Oxford handbook of human motivation, 85-107.

Eccles, J., \& Wang, M.T. (2012). Part I Commentary: so what is student engagement anyway?. Em S.L. Christenson, A.L. Reschly, \& C. Wylie (Orgs.), Handbook of research on student engagement (pp. 133-145). New York: Springer Sciences.

Fior, C.A., Mercuri, E., \& Silva, D. (2013). Evidências de validade da Escala de Envolvimento Acadêmico para universitários. Avaliação Psicológica, 12 (1), 81-89.

Guimarães, S.É.R. \& Bzuneck, J.A. (2008). Propriedades psicométricas de um instrumento para avaliação da motivação de universitários. Ciências \& Cognição, 13(1), 101-113.

Instituto Nacional de Estudos e Pesquisas Educacionais Anísio Teixeira [INEP] (2014). Matrículas no ensino superior crescem 3,8\%. Recuperado: 17 dez. 2014. Disponível:http://portal.inep. gov.br/visualizar/-/asset_publisher/6AhJ/content/matriculas-noensino-superior-crescem-3-8.

Joly, M.C.R.A. \& Prates, E.A.R. (2011). Avaliação da Escala de Motivação Acadêmica em estudantes paulistas: propriedades psicométricas. Psico-USF, 16 (2), 175-184.

Leal, E.A., Miranda, G.J., \& Carmo, C.R.S. (2013). Teoria da autodeterminação: uma análise da motivação dos estudantes do curso de ciências contábeis. Revista Contabilidade \& Finanças, 24 (62), 162-173.

Mercuri, E. \& Fior, C. (2012). Análise dos fatores preditivos da evasão em uma universidade confessional. Em II Conferencia
Latinoamericana sobre el abandono de la educación superior (Org.), Libro de actas (pp. 178-189). Rio Grande do Sul: CLABES.

Reeve, J. (2012). A self-determination theory perspective on student engagement. Em S.L. Christenson, A.L. Reschly, \& C. Wylie (Orgs.), Handbook of research on student engagement (pp. 149172). New York: Springer US.

Rodrigues, N.R.N.M., Silva, A.F., Oliveira, M.S., Rodrigues, A.G., Souza, A.M., Lopes, M., Soares, R.P.O., \& Tostes, M.E.L. (2012). Alunos Ingressantes nas Engenharias e a Aprendizagem Básica em Matemática [Resumo]. Em XL Congresso Brasileiro de Educação em Engenharia (Org.), Anais do XL Congresso Brasileiro em Engenharia. Belém-Pará: ABENGE.

Ryan, M.R. \& Deci, E.L. (2000). Intrinsic and Extrinsic Motivations: Classic Definitions and New Directions. Contemporary Educational Psychology, 25(1), 54-67.

Santos, A.A.A., Mognon, J.F., de Lima, T.H., \& Cunha, N.B. (2011). A relação entre vida acadêmica e a motivação para aprender em universitários. Psicologia Escolar e Educacional, 15 (2), 283-290.

Silva, S.A.P.S., Melo, L.F., Nunes, H.C.B., \& Moura, S. (2012). Atividades acadêmico-científico-culturais na formação do profissional de Educação Física. Motriz: Revista de Educação Física, 18(1), 92-103.

Traina-Chacon, J.M., \& Calderón, A.I. (2015). Aexpansão da educação superior privada no Brasil: do governo de FHC ao governo de Lula. Revista Iberoamericana de Educación Superior, 6(17), 78-100.

Vansteenkiste, M., Lens, W., \& Deci, E.L. (2006). Intrinsic Versus Extrinsic Goal Contents in Self-Determination Theory: Another Look at the Quality of Academic Motivation. Educational Psychologist, 4(1), 19-31.

Recebido em: 03 de agosto de 2016 Aprovado em: 24 de novembro de 2016

\section{Sobre as autoras}

Rebeca Cruz Porto (rebeca.c.porto@hotmail.com).

Graduada em Psicologia pela Universidade Federal do Vale do São Francisco-Petrolina - PE (Univasf). Mestranda do Programa de Pós-graduação em Psicologia da Univasf.

Marina Pereira Gonçalves (marina.goncalves@univasf.edu.br)

Doutora em Psicologia Social pela UFPB. Docente do curso de Psicologia da Universidade Federal do Vale do São Francisco - Petrolina - PE (Univasf), docente permanente do Programa de Pós-graduação em Psicologia da Univasf e docente colaboradora do Programa de Pós-graduação em Educação Física da Univasf. 